\title{
Types and frequency of ocular abnormalities in stroke patients in university of Calabar teaching hospital, Calabar, Nigeria
}

\begin{abstract}
Aim: The study was done to identify the types and determine the frequency of ocular abnormalities in patients diagnosed with stroke in University of Calabar Teaching Hospital. It is a hospital based observational study where all consecutive stroke patients, both old and new cases who met inclusion criteria were examined for ocular abnormalities. Data were collected and analyzed with SPSS 20. Descriptive statistics (frequencies, percentages, charts, means and standard deviation) were used to summarize the variables.
\end{abstract}

Results: A total of 760 eyes of 380 patients were examined. The mean age was 59.5 years \pm 11.1 with age range 18 to 87 years. There were 197 males $(51.8 \%)$ and 183 $(48.2 \%)$ females. The frequency of ocular abnormalities was $91.1 \%$. The most common ocular abnormalities were hypertensive retinopathy $(30 \%)$, lens opacities $(29.7 \%)$, ocular surface disorder (17.1\%), facial nerve palsy (15.5\%) and glaucoma $(15.5 \%)$.

Conclusion: The frequency of ocular abnormalities and visual impairment is higher in stroke patients than in the general population; though the author recognizes that hospital based studies may not fully represent the true picture of the general population. The abnormalities can worsen the associated morbidity of stroke especially as many of the stroke patients are not aware of these ocular abnormalities. Routine ocular evaluation should be included in the clinical evaluation of stroke patients. Identification and management of the ocular abnormalities could significantly reduce their morbidity
Volume 9 Issue 2 - 2019

\author{
Kennedy U Nwachukwu,' Affiong A Ibanga,' \\ Bassey A Etim,' Ekanem Philip-Ephraim² \\ 'Department of Ophthalmology, University of Calabar Teaching \\ Hospital, Nigeria \\ ${ }^{2}$ Department of Internal Medicine, University of Calabar \\ Teaching Hospital, Nigeria
}

Correspondence: Ekanem Philip-Ephraim, Department of Internal Medicine, University of Calabar Teaching Hospital, Calabar, Email nneninge@yahoo.com

Received: February 16, 2019 | Published: March I5, 2019

Keywords: ocular abnormalities, stroke, Calabar, Nigeria

\section{Introduction}

Stroke is defined as "rapidly developing clinical signs of foca (or global) disturbance of cerebral function, with symptoms lasting 24 hours or longer or leading to death, with no apparent cause other than of vascular origin". ${ }^{1}$ It is also referred to as Acute Brain Insult. Recent researches suggest a new definition which will not have a time criterion but rather will be based on tissue damage. ${ }^{2}$ However, stroke is a clinical diagnosis and the above definition remains acceptable by World Health Organization (WHO).

Stroke, a leading cause of morbidity and mortality worldwide, is likely to worsen in developing countries over the next two decades based on projections by the World Health Organization. ${ }^{3}$ More than 30 million people worldwide are estimated to have stroke, of which about 12 million live with moderate to severe disabilities. ${ }^{4}$ The incidence of stroke is significant with varying figures across the globe and even within the same region. Stroke incidence reports ranged from 76 per 100000 population per year in Australia to 119 per 100 000 population per year in New Zealand, after adjusting to the WHO world population. ${ }^{5}$ A meta-analysis of stroke epidemiology in Africa, also shows a wide variation in the incidence and prevalence of stroke across Africa. ${ }^{6}$ In developed countries like the United States, stroke is the most common cause of disability and the fifth most common cause of death. ${ }^{7}$ The major disabilities are due to hemiparesis/hemiplegia, visual dysfunction, speech and hearing difficulties. ${ }^{8}$ There is paucity of data on the contribution of visual dysfunction to stroke disabilities in Africa.

In Nigeria, the prevalence of stroke is estimated at 1.14 per 1000 , while the 30 -day case fatality rate is as high as $40 \%,{ }^{9}, 10$ A community based study in the middle-belt of Nigeria found a stroke prevalence of 1.31 per 1000 population. ${ }^{11}$ Stroke has been found to be the most common neurological cause of hospital visits in Nigeria, accounting for up to $42 \%$ of the neurological admissions ${ }^{12}$ and $52.5 \%$ of neurological emergencies. ${ }^{13}$ The high morbidity and mortality rates usually provoke significant degree of anxiety in people with risk factors to stroke, affected patients and even care-givers.

The eye and the brain share several features in common especially as both develop from the diencephalon. The ophthalmic artery (blood supply to the eye) is a branch of the internal carotid artery, which is the main blood supply to the brain. There are 12 cranial nerves; half of which have close association with the eye and its adnexae. Many parts of the brain are involved in the processing and control of visual function. The primary visual area known as Brodman area 17 is in the striate cortex, and is located in the occipital lobe of the brain. ${ }^{14}$ The surrounding areas 18 and 19 known as Visual Association areas mediate other aspects of the visual function. The frontal lobe of the brain initiates voluntary saccades, the temporal lobe is involved in visual memory, while the parietal lobe and the midbrain control and coordinate eye movements. It has been suggested that about $30 \%$ of neurons in the cerebral cortex are devoted to vision..$^{15}$ The optic nerves connect the eye to the brain, also sharing the meningeal coverings of the brain. ${ }^{16}$ Most of the anterior visual pathway lies in contact with brain substance, while the posterior visual pathway is in the substance of the brain. These close associations therefore suggest that pathology of the brain will affect the eye more than any other organ and ocular features of stroke may be more common than previously acknowledged.

There are many documented ocular findings seen in patients with stroke, ranging from minor and asymptomatic conditions to severe debilitating ones. The most disturbing is sudden complete loss of 
vision or visual field loss. ${ }^{17,18}$ Others include, drooping of eyelids, disorders of ocular motility, gaze palsies, diplopia and nystagmus. ${ }^{8}$ To the best of our knowledge, no study of this nature has been carried out among stroke patients in this part of Nigeria. This study therefore sets out to highlight ocular abnormalities in stroke patients as seen in the University of Calabar teaching Hospital (UCTH), Calabar, Nigeria.

\section{Methods}

This study is a hospital based observational cross-sectional clinical study. All old and newly diagnosed stroke patients, 18 years and above who have been managed or are being managed by the Neurology Unit, Department of Internal Medicine, UCTH were eligible to be recruited into the study after their consent. Old patients were recruited from clinic visits. New patients were recruited from the clinic, the accident and emergency unit and from the medical wards after admission. The recruitment was from January to October 2016. Non-probability serial recruitment method was used. Subjects were identified with serial numbers and their hospital numbers, and their hospital folders marked to avoid being included more than once in the study. Approval for the study was given by the Health Research Ethics Committee of UCTH. Written and verbal informed consent was obtained from subjects by the principal investigator.

Distance and near visual acuity (VA) were measured using the Snellen's or E-chart and Near-chart respectively. A pin-hole VA was done when presenting VA was 6/18 or worse and patient could understand and cooperate with the test. Confrontational visual field assessment was carried out from a distance of one metre. Subjects were examined for facial asymmetry especially from facial nerve palsy. The eyelids were evaluated for ptosis by using the MarginReflex-Distance (MRD-1). Skew deviation and ocular mal-alignment was looked out for using the Hirschberg test. Ocular motility tests were done by moving a fingers in the diagnostic positions of gaze. Vergence and duction tests were done to check for gaze palsies and cranial nerve palsies.

The ocular surface and anterior segment was examined with a bright penlight for ocular surface irregularities and corneal clarity. The anterior chamber depth was assessed using the eclipse sign and pupillary light reflexes also assessed. The intraocular pressure was checked with a Perkins handheld tonometer. The Perkins tonometer head was disinfected with $75 \%$ methylated spirit swabs and wiped with normal saline swab between patients.

The subjects' pupils were dilated with Trophen eye drops (ARISTOPHARMA LTD; batch No. 15J06), a combination of 5\% phenylephrine and $0.8 \%$ tropicamide prior to fundoscopy. This was done by a nurse. One drop was instilled into each eye and the subject asked to close the eyes to reduce systemic absorption. Most of the subjects' pupils were adequately dilated in $10-15$ minutes after only one drop. Some patients needed a second instillation.

A dilated direct fundoscopy (with Keeler Professional ophthalmoscope) was done on all subjects. Media opacities especially from lens opacities were assessed by observing the red reflex with the ophthalmoscope dialed to +10 dioptre, from a distance of about one foot. The optic disc was assessed for signs of neuropathy and disc edema. The retina was studied in quadrants, following the vascular arcades, looking out for retinal haemorrhages, cotton wool spots, focal retinal narrowing, arteriovenous nipping and any other lesion. Further examinations were carried out according to individual needs and for the purpose of management.
Data analysis: Data from the ocular evaluation form (appendix two) were inputted into statistical package for social sciences (SPSS) software for Windows version 20 (SPSS inc., Chicago, IL, USA) and analyzed using same. Descriptive statistics (frequencies, percentages, charts, means and standard deviation) were used to summarize the variables.

\section{Results}

A total of 760 eyes of 380 stroke patients were examined. The mean age of the subjects was 59.5 years \pm 11.1 and age range 18 to 87 years. There were 197 males (51.8\%) and 183 females (48.2\%). The mean age was $60.2 \pm 11.1$ for males and $58.7 \pm 11.1$ for females. There was no statistical difference between the two, $t(378)=1.352$, $p$-value $=0.177$.

\section{Ocular findings}

\section{Visual acuity and causes of visual impairment}

Using presenting visual acuity (PVA), 9\% of eyes had normal vision, $38.9 \%$ had mild visual impairment, $27.8 \%$ had moderate visual impairment, $1.8 \%$ had severe visual impairment and $12.1 \%$ were blind (Table 1). A total of 317 eyes $(41.7 \%)$ had visual acuity worse than $6 / 18$. Visual acuity was not done in 39 subjects who were either unconscious or unable to cooperate.

Table I Presenting Visual Acuity frequency in stroke patients in UCTH

\begin{tabular}{|c|c|c|c|c|c|}
\hline \multirow{2}{*}{ Grading (Total \%) } & \multirow{2}{*}{ Visual Acuity } & \multicolumn{2}{|c|}{ Right eye } & \multicolumn{2}{|c|}{ Left eye } \\
\hline & & No. & (\%) & No. & (\%) \\
\hline \multirow{3}{*}{ Normal VA (9\%) } & $\begin{array}{l}\text { not possible/not } \\
\text { done }\end{array}$ & 39 & $(10.3)$ & 39 & $(10.3)$ \\
\hline & $6 / 6$ & 34 & $(8.9)$ & 35 & $(9.2)$ \\
\hline & $6 / 9$ & 58 & $(I 5.3)$ & 53 & (I3.9) \\
\hline \multirow{3}{*}{$\begin{array}{l}\text { Mild visual impairment } \\
\text { (38.94\%) }\end{array}$} & $6 / 12$ & 58 & $(15.3)$ & 58 & $(I 5.3)$ \\
\hline & $6 / 18$ & 35 & $(9.2)$ & 34 & $(8.9)$ \\
\hline & $6 / 24$ & 42 & (II.I) & 38 & $(10.0)$ \\
\hline \multirow[t]{2}{*}{$\begin{array}{l}\text { Moderate visual } \\
\text { impairment (27.76\%) }\end{array}$} & $6 / 36$ & 34 & $(8.9)$ & 43 & $(I I .3)$ \\
\hline & $6 / 60$ & 27 & (7.I) & 27 & (7.I) \\
\hline \multirow[t]{3}{*}{$\begin{array}{l}\text { Severe visual } \\
\text { impairment ( } \mathbf{I . 8 4 \% )}\end{array}$} & $5 / 60-3 / 60$ & 9 & $(2.4)$ & 5 & $(1.3)$ \\
\hline & $2 / 60-1 / 60$ & 15 & (3.9) & 21 & $(5.5)$ \\
\hline & $\mathrm{CF}$ & 6 & $(1.6)$ & 8 & $(2.1)$ \\
\hline \multirow[t]{4}{*}{$\begin{array}{l}\text { Blindness } \\
\text { (12.1 I\%) }\end{array}$} & $\mathrm{HM}$ & 10 & $(2.6)$ & 5 & $(\mathrm{I} .3)$ \\
\hline & PL & 3 & $(0.8)$ & 5 & $(1.3)$ \\
\hline & NPL & 10 & $(2.6)$ & 9 & $(2.4)$ \\
\hline & Total & 380 & $(100.0)$ & 380 & $(100.0)$ \\
\hline
\end{tabular}

\section{Causes of visual impairment}

The common ocular abnormalities seen in subjects with mild visual impairment were hypertensive retinopathy $(26.5 \%)$, ocular surface disorder $(11.9 \%)$ and refractive error $(9.3 \%)$, while in subjects with moderate visual impairment, they were age related maculopathy $(14.6 \%)$, hypertensive retinopathy $(13.6 \%)$, diabetic retinopathy $(12.6 \%)$, and cataract (10.7\%). Severe visual impairment was recorded in at least one eye of 9 subjects, of which two $(22.2 \%)$ had cataract. Other abnormalities were as shown in Table 2. The major causes of blindness in subjects included glaucoma (36.4\%), cataract (18.2\%), other optic neuropathy (13.6\%) and age related maculopathy (4.5\%). 


\section{Ocular abnormalities}

The frequency of ocular abnormalities in the study population was $91.1 \%$. The commonest ocular abnormalities found in the study population were cataract, seen in $133(35 \%)$ subjects and hypertensive retinopathy recorded in $115(30.3 \%)$ subjects. There were 169 (44.5\%) subjects who had more than one ocular abnormality, and as such multiple entries. There were more posterior segment abnormalities than anterior segment abnormalities. A hundred and sixty-four (164) $(43.2 \%)$ subjects had only posterior segment abnormalities, 65 (17.1\%) had only anterior segment abnormalities while 117 (30.8\%) subjects had ocular abnormalities in both anterior and posterior segments. Ocular abnormalities were absent in 34 (8.9\%) subjects.

\section{Anterior segment abnormalities}

Cataract was the most common anterior segment abnormality. The subjects who had cataract consisted of $113(29.7 \%)$ with mild to moderate but visually significant lens opacities and $20(5.3 \%)$ subjects with dense cataract. Others are as shown in Table 3. The anterior segment neuro-ophthalmic abnormalities that may be related to the stroke event include facial nerve palsy, disorders of ocular motility, ptosis, third/sixth cranial nerve palsies and lid retraction (Table 3).

Table 2 Causes of visual impairment in stroke patients in UCTH.

\begin{tabular}{llccc}
\hline Ocular abnormalities & Mild visual impairment & Moderate visual impairment & Severe visual impairment & Blindness \\
\hline HTN RET. & $40(26.5 \%)$ & $14(13.6 \%)$ & - & $2(4.5 \%)$ \\
Cataract & $1(0.7 \%)$ & $11(15.5 \%)$ & $2(22.2 \%)$ & $8(18.2 \%)$ \\
Glaucoma & $13(8.6 \%)$ & $6(5.8 \%)$ & $1(11.1 \%)$ & $16(36.4 \%)$ \\
OON & - & $2(1.9 \%)$ & $1(11.1 \%)$ & $6(13.6 \%)$ \\
DM RET. & $10(6.6 \%)$ & $13(12.6 \%)$ & $1(11.1 \%)$ & $1(2.3 \%)$ \\
ARM & $7(4.6 \%)$ & $15(14.6 \%)$ & - & - \\
Ref. error & $14(9.3 \%)$ & $7(6.8 \%)$ & $1(1.11 \%)$ & $2(4.5 \%)$ \\
RAO & - & $1(1.0 \%)$ & - & - \\
OSD & $18(11.9 \%)$ & $3(2.9 \%)$ & & -
\end{tabular}

HTN RET, hypertensive retinopathy; OON, other optic neuropathy; DM RET, diabetic retinopathy; ARM, age related maculopathy; Ref. error: refractive error; RAO, retinal artery occlusion; OSD, ocular surface disorder

Table 3 Ocular abnormalities in stroke patients in UCTH

\begin{tabular}{llll}
\hline Ocular abnormalities anterior segment & Frequency (\%) & Ocular abnormalities posterior Segment & Frequency (\%) \\
\hline Mild to moderate lens opacities & $113(29.7)$ & Hypertensive Retinopathy & $115(30.3)$ \\
Ocular surface disorder & $65(17.1)$ & Glaucoma & $55(14.5)$ \\
Facial nerve palsy & $59(15.5)$ & Diabetic retinopathy & $50(13.2)$ \\
Refractive error & $51(13.4)$ & Age Related Maculopathy & $43(11.3)$ \\
Disorders of ocular motility & $46(12.1)$ & Retinal arteriosclerosis & $33(8.7)$ \\
Dense cataract & $20(5.3)$ & Disc edema/papilloedema & $32(8.4)$ \\
Ptosis & $17(4.5)$ & Other retinopathy/detachment & $29(7.6)$ \\
Third nerve/Sixth nerve palsy & $7(1.8)$ & Retinal vein occlusion & $26(6.8)$ \\
Lid retraction & $4(1.1)$ & Other optic neuropathy & $19(5.0)$ \\
& & Retinal artery occlusion/embolus & $10(2.6)$ \\
\end{tabular}

\section{Posterior segment abnormalities}

The most common posterior segment abnormality was hypertensive retinopathy $(30 \%)$. Others included glaucoma $(14.5 \%)$, diabetic retinopathy $(13.2 \%)$, age related maculopathy $(11.3 \%)$, and retinal arteriosclerosis $(8.7 \%)$ as shown in the Table 3.

\section{Types of disorders of ocular motility}

There were four types of disorders of ocular motility observed in subjects (Figure 1). Horizontal gaze palsy was the commonest disorder of ocular motility observed, while skew deviation was the least common. These disorders of ocular motility were not classified into sub-types.

\section{Neuro-Ophthalmic abnormalities}

Table 4 shows that the most common neuro-ophthalmic manifestations in subjects were facial nerve palsy (15.5\%) and disorders of ocular motility (12.1\%), while lid retraction was the least $(1.1 \%)$.

\section{Hypertensive and diabetic retinopathy}

There were 115 (30.3\%) subjects showing features of hypertensive retinopathy. Grading was done according to modified Scheie classification. ${ }^{19}$ Subjects showing features of Grade 2 hypertensive retinopathy were the highest $(53 \%)$, followed by grade $3(19.1 \%)$, grade $4(14.8 \%)$ and grade $1(13 \%)$, as shown in Figure 2. Diabetic 
retinopathy was found in 50 (13.2\%) subjects; 45 subjects (90\%) had non-proliferative diabetic retinopathy while $5(10 \%)$ subjects had proliferative diabetic retinopathy.

Disorders of ocular motility

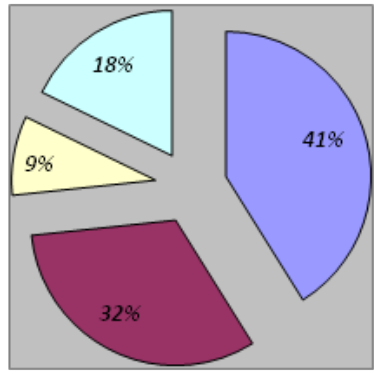

\begin{tabular}{|c|}
\hline $\begin{array}{c}\text { Horizontal } \\
\text { gaze palsy } \\
\square \text { Vertical gaze } \\
\text { palsy }\end{array}$ \\
$\square$ Skew \\
deviation \\
$\square$ Nystagmus
\end{tabular}

Figure I Types of disorders of ocular motility in stroke patients in UCTH. Frequency

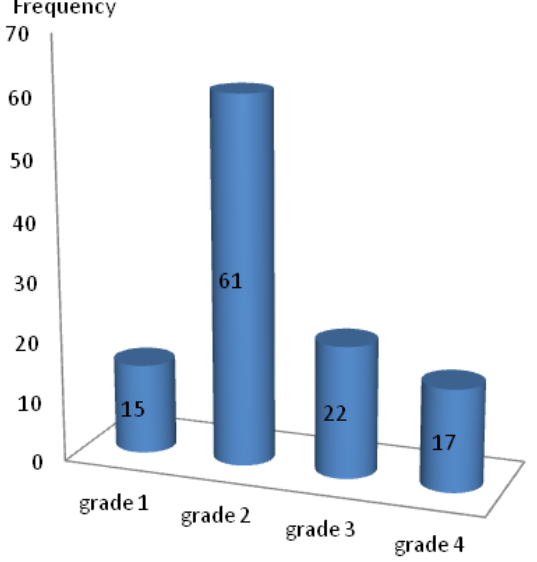

- Hypertensive retinopathy

Figure 2 Hypertensive retinopathy grading in stroke patients in UCTH.

\section{Glaucoma}

Glaucoma was present in 55 (14.5\%) subjects; 27 (49.1\%) had high intraocular pressures (IOP), another 27 (49.1\%) had normal IOP and one of them didn't have IOP measured (poor cooperation). Twenty seven subjects $(57.4 \%)$ who had high IOP were diagnosed with glaucoma while $26(9.1 \%)$ of those who had normal IOP were diagnosed with glaucoma. Glaucomatous discs were more prevalent in subjects with high IOP (p-value: $<0.001$ ).

\section{Cortical visual impairment and homonymous hemianopia}

There were 21 subjects diagnosed with cortical visual impairment. Computerized tomography scan showed occipital lesions in 7 $(33.3 \%)$, parietal lesions in $2(9.5 \%)$, lacunar infarct in $1(4.8 \%)$ and multiple infarcts in 1 subject. There were no brain imaging results for $10(47.6 \%)$ of these subjects. Homonymous hemianopic visual field defect on confrontational testing was found in $9(42.9 \%)$ of these subjects with homonymous hemianopia. Most of the subjects who were sent for automated visual field testing could not do the test due to either physical disabilities or financial constraint. The few that did the automated test had unreliable visual field print-outs

\section{Other retinal abnormalities in subjects}

The most common retinal abnormality found was focal arteriolar narrowing in 115 (30.3\%) subjects, followed by hard exudates in 108 $(28.5 \%)$ subjects, arterio-venous nipping in $90(23.7 \%)$ and retinal haemorrhages in $89(23.5 \%)$ subjects. Pigmentary retinopathy seen in $47(12.4 \%)$ subjects was the least (Figure 3).

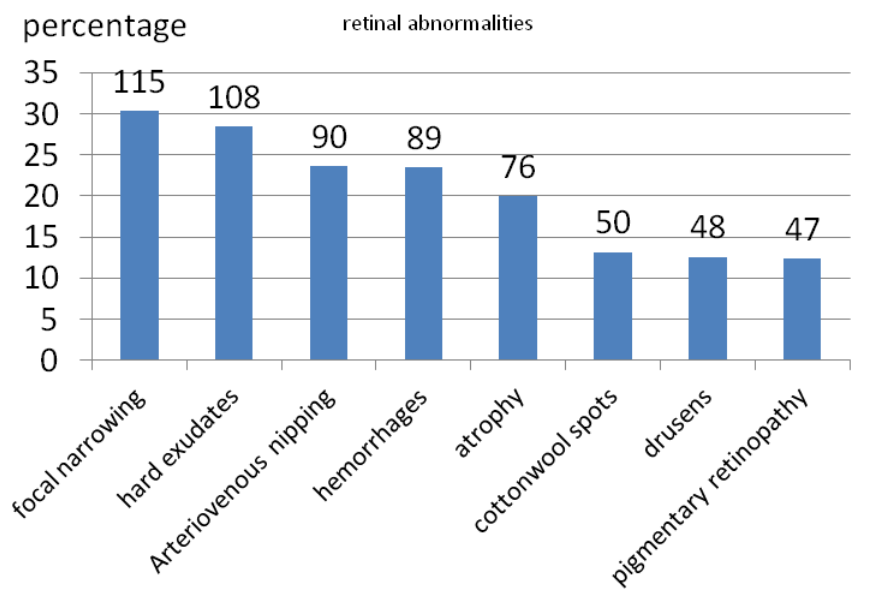

Figure 3 Types of retinal abnormalities among stroke patients in UCTH. Table 4 Neuro-ophthalmic abnormalities in stroke subjects in UCTH

\begin{tabular}{ll}
\hline Neuro-ophthalmic abnormality & Frequency (\%) \\
\hline Facial nerve palsy & $59($ I5.5) \\
Disorders of ocular motility & $46(12.1)$ \\
Cortical visual impairment & 2 I 5.5) \\
Ptosis & $17(4.5)$ \\
Homonymous hemianopia & $14(3.7)$ \\
Third nerve/sixth nerve palsy & $7(1.8)$ \\
Lid retraction & $4(I . I)$
\end{tabular}

\section{Ocular complaints and ocular abnormalities}

Among the 346 subjects who had ocular abnormalities on examination, $123(35.5 \%)$ of them did not have any ocular complaint, $94(27.2 \%)$ complained of moderate bilateral blurring of vision, 41 $(11.8 \%)$ had severe unilateral blurring of vision while 31 (9\%) had gritty sensation. Other complaints included severe bilateral visual loss in $27(7.8 \%)$ subjects, loss of central vision in $17(4.9 \%)$, double vision in $7(2 \%)$, ocular deviation in $5(1.4 \%)$ and floaters in one $(0.3 \%)$ subject. Most of the subjects; 28 out of 34 (84.4\%), with no identified ocular abnormality had no complaint, $5(14.7 \%)$ of them complained of moderate blurring of vision while one (2.9\%) complained of ocular pains.

\section{Major ocular abnormalities in subjects without complaints}

There were 151 subjects who did not have ocular complains; $123(81.5 \%)$ had ocular abnormalities while $28(18.5 \%)$ of them did not have. The ocular abnormalities found included hypertensive retinopathy, facial nerve palsy, disorders of ocular motility and few others. Other relevant information about this group include; 29 $(19.2 \%)$ of these subjects had VA less than $6 / 18$ in at least one eye, $30(19.9 \%)$ had cognitive impairment and $25(16.6 \%)$ of them were aphasic. 


\section{Discussion}

Stroke morbidity is largely caused by persistent neurologic deficits which may be motor, sensory or both. Disabilities may be further compounded by visual dysfunction or ocular abnormalities which are often times ignored probably because routine ocular examination is not the norm during evaluation in stroke patients. Using presenting visual acuity (PVA), $28.4 \%$ of eyes had moderate visual impairment, $1.8 \%$ had severe visual impairment, and $12.1 \%$ were blind. A total of 311 eyes $(40.9 \%)$ had a PVA of less than 6/18. Olubor et al. reported $9.5 \%$ for moderate visual impairment, $14.5 \%$ for severe visual impairment, $8.2 \%$ for blindness, with $32.4 \%$ of them having PVA of $<6 / 18$. The frequency of blind eyes in the index study $(12.1 \%)$ is higher than that of Olubor $(8.2 \%)$ probably because of differences in attitude towards eye care and blindness between the sample populations. These values are significantly higher than the prevalence reported in the Nigeria National Blindness and Visual Impairment study ${ }^{20}(4.2 \%$ among those 40 years and above) and suggests that blindness prevalence is higher among stroke patients than in the general population. The author however recognizes that the index study is hospital based and not easily comparable with population based studies.

The frequency of all ocular abnormalities in the index study was $91.1 \%$. This high frequency can largely be attributed to the fact that ocular abnormalities and visual impairment are higher in the elderly ${ }^{20}$ who make up majority of stroke sufferers. This frequency is lower but close to that of the study done in UBTH $(95.5 \%)$. The study in UBTH had patients within the age range of 43 to 88 years (excluding more of the younger age group) while the index study had age range 18 to 87 years. Since most of the ocular abnormalities have been attributed to aging, this difference in age range could be the reason why the UBTH study had a higher frequency of ocular disorders.

The most common ocular abnormality observed was lens opacities. This is a similar finding in the study done in UBTH by Olubor et al which also recorded lens opacities as the most common ocular abnormality in stroke patients. This is due to the fact that the study population consists mainly of the middle aged and elderly where the occurrence of cataract is known to be more common. However the frequency in the UBTH study is much higher (71.8\%) probably because all identified lens opacities were included regardless of visual significance. In the index study only lens opacities which caused symptoms or reduction of vision as defined in the methodology were considered visually significant and thus recorded.

Ocular surface disorders were common in study subjects, with a frequency of $17.1 \%$. This differs from the study done by Olubor et $\mathrm{al}^{21}{ }^{21}$ which reported $12.4 \%$ frequency for pterygium. The index study included pterygium, pingueculum, conjunctival concretions and other surface irregularities which likely resulted to a higher frequency than that of Olubor et al, ${ }^{21}$ which reported only pterygium.

Horizontal gaze palsy was the most common disorder of ocular motility observed in subjects, making up $41 \%$ of disorders of ocular motility observed. The brainstem was more commonly involved in all the types of ocular motility disorders, but the parietal area was more involved in the subjects with horizontal gaze palsy. Other affected areas included the occipital, frontal, and cerebellar areas. This picture may not be a true representation of the target population because brain imaging results were available only in $110(28.9 \%)$ subjects. The brain areas affected in stroke patients who had disorders of ocular motility has been documented in some studies with varying patterns.
In a prospective multicenter cohort trial on stroke patients carried out by Rowe et al., ${ }^{22}$ where $54 \%$ of participants had ocular motility disorders, frequently involved brain areas were the cerebellum, the brainstem and diencephalic areas. Other brain areas involved included the parietal, occipital and temporal lobes.

Facial nerve palsy was seen in $15.5 \%$ of study subjects. No study on prevalence of facial nerve palsy in stroke patients was found, but few studies reported a prevalence of less than $0.2 \%$ in the general population. ${ }^{23}$ Ptosis was seen in 17 (4.5\%) patients; ten of them were aponeurotic ptosis, five were secondary to third nerve palsy while two of them had mechanical causes. Olubor et al found ptosis in $2.9 \%$ of his study population, which is lower than that reported in the index study. Most of the ptosis observed in the index study were not of neurologic aetiology, which could account for the higher frequency. Cerebral ptosis is said to be rare in most literature but a study done by Averbuch-Heller et al. ${ }^{24}$ in Israel reported a high frequency of $37.5 \%$ despite a small sample size of 64 stroke subjects. A plausible reason could not be given for this. Lid retraction was seen in $4(1.1 \%)$ patients of whom one was on treatment for hyperthyroidism. Studies on the prevalence of lid retraction in stroke patients were not found.

Hypertensive retinopathy was the most common posterior segment abnormality, and the second common ocular abnormality observed in the index study. Out of the 291 recorded hypertensive subjects, 106 (36.4\%) had hypertensive retinopathy. Similar frequency was reported in a study done in 213 hypertensive patients in a teaching hospital in Iran, where $39.9 \%$ of them had hypertensive retinopathy. ${ }^{25}$ Olubor et al. ${ }^{21}$ and Abah et al. ${ }^{26}$ in their studies did not report hypertensive retinopathy as an entity; rather they reported retinal features like arterio-venous nipping, retinal haemorrhages, cotton wool spots, and exudates as separate entities. If those signs (which are common features of hypertensive retinopathy) are put together, the frequency of hypertensive retinopathy would likely be comparable to the index study. The prevalence of hypertensive retinopathy in stroke may even be higher than reported here because some signs of hypertensive retinopathy are transient, for example flame-shaped haemorrhages and cotton wool spots. Again, the presence of media opacities may obscure these features during fundoscopy.

The frequency of glaucoma in the index study was $14.5 \%$, similar to the $14.2 \%$ reported by Olubor et al. ${ }^{21}$ This suggests that glaucoma prevalence is higher in stroke patients when compared with values in the Nigeria National Blindness and Visual Impairment Study. This can be attributed to the fact that glaucoma is a progressive condition and its prevalence increases with increasing age (an important risk factor of stroke).

Cortical Visual Impairment was diagnosed in 21 (5.5\%) subjects; 7 $(33.3 \%)$ had lesions affecting the occipital lobe and $2(9.5 \%)$ subjects had infarcts affecting the parietal lobe. Ten $(47.6 \%)$ of them did not have brain imaging done due to financial constraints. There was homonymous hemianopia on confrontational testing in $9(42.9 \%)$ of these subjects with cortical visual impairment. In the whole study population, homonymous visual field defects were found in 14 subjects, with 5 of them having their occipital areas affected. One person had a lacunar infarct while others didn't have brain imaging done. Thus it is suggestive, though not conclusive that occipital area (which contains the primary visual cortex (Brodman area 17) where visual pathway nerve fibers converge and visual impulses from the retina are resolved) is the most commonly affected area in subjects with cortical visual impairment and homonymous hemianopia. 
The finding would have been more conclusive if brain imaging results were available in all subjects. In a prospective multi-centre observational case cohort study done by the Vision In Stroke group in United Kingdom, about $50 \%$ of selected subjects had visual field loss due to cortical visual impairment. ${ }^{27}$ The study population included only stroke patients suspected of having visual difficulty, so the higher prevalence is expected.

The commonest retinal abnormality was focal arteriolar narrowing $(30.3 \%)$, followed by hard exudates $(28.5 \%)$. This does not agree with the studies mentioned earlier. For example, Olubor et al. ${ }^{21}$ reported focal arteriolar narrowing in $2.4 \%$ of subjects, hard exudates in $7.6 \%$ and generalized narrowing in $24.7 \%$. The wide variations could be due to inter-observer variations in defining vascular and retinal pathology. Index study recorded retinal hemorrhages in $23.5 \%$ of subjects while Da Silva et al..$^{28}$ Abah et al ${ }^{26}$ and Olubor et al. ${ }^{21}$ recorded it in $27 \%, 16.5 \%$ and $10.6 \%$ respectively. Retinal hemorrhages would be expected to be more in the patients with recent or acute stroke (less than 1 year duration) examined in these three studies, compared with the index study where a significant percentage $(43.2 \%)$ of subjects had stroke of long standing duration (more than 1 year). The subjects with stroke of longer duration would be expected to have better control of risk factors (especially hypertension) and less of retinal haemorrhages. However, this is an assumption and long standing stroke patients may not control their stroke risk factors despite clinic visits, thus presenting with recurrent retinal haemorrhages. Only Da Silva recorded a higher prevalence of retinal haemorrhages in the study population of acute stroke patients. No plausible explanation can be suggested at the moment why retinal haemorrhages would be less prevalent in more acute stroke patients with similar risk factors of hypertension and diabetes mellitus.

The index study found age related maculopathy in $43(11.3 \%)$ subjects. This differs from the studies carried out by Da Silva et al. ${ }^{28}$ and $\mathrm{Abah}$ et $\mathrm{al}^{26}$ which reported age related maculopathy as the most common retinal abnormality in stroke (30\% and $36.7 \%$ respectively). The age range and mean ages in these studies were similar to index study. Da Silva et al carried their study in a Singapore population where there is a higher prevalence of age related maculopathy than in blacks. ${ }^{29,30}$ The finding of age related maculopathy as the commonest finding in a black population of stroke patients as reported by Abah et al. ${ }^{26}$ could not be easily explained without further investigations, though it may be accounted for by differences in definition of terms.

In the index study $35.5 \%$ of subjects who had ocular abnormalities did not have any ocular complaint at the time of evaluation. In the Vision In Stroke study, $10 \%$ of subjects who had documented visual field loss were not aware of the defect and did not have any symptom. ${ }^{27}$ This indicates that many stroke patients with ocular abnormalities are not aware of the problems, so routine ocular evaluation in these patients would help identify visual threatening conditions and possibly prevent blindness. The prevalence of ocular abnormalities in the subjects who did not have complaints was $81.5 \%$. Their inability to complain may be related to significant disabilities like aphasia and cognitive impairment found among them. Other reasons were asymptomatic ocular abnormality and unilateral loss of vision of which the subjects were not aware of.

Diabetic retinopathy is an ocular complication seen in diabetes mellitus. The prevalence of diabetic retinopathy in index study was $13.2 \%$; $90 \%$ of them had non-proliferative diabetic retinopathy (NPDR) while $10 \%$ had proliferative diabetic retinopathy.

\section{Limitations of the study}

Facilities were not available to obtain a fundus angiogram in some patients who had retinal vascular abnormalities of uncertain cause thus limiting definitive diagnosis in those patients. Assessment of the pupillary near reflex was omitted in the study evaluation form and was not performed. So abnormalities of the pupillary near reflex which occur in dorsal column lesions may have been missed. There were significant challenges in obtaining reliable automated visual field results in stroke patients. These included poor reliability indices like high fixation errors, cognitive deficits in some subjects, and difficulties in conveying subjects with hemiparesis to perimetry room.

\section{Conclusion}

The frequency of ocular abnormalities is very high in stroke patients. These ocular abnormalities ranged from mild conditions of little or no visual significance like mild ocular surface irregularities, to visually debilitating ones like end-stage glaucoma and retinal artery occlusions. The frequency of visual impairment is higher in stroke patients than in the general population of similar age group, though the fact that the subjects were sick patients seen in a hospital setting introduces a bias and the results may not be a true representation of the population. These ocular abnormalities can worsen the associated morbidity of stroke especially as many of the stroke patients are not aware of these ocular abnormalities.

\section{Recommendation}

i. The neurology unit in conjunction with ophthalmology departments of hospitals should develop a protocol for routine visual function assessment in order to identify stroke patients that have conditions which can worsen their disabilities or morbidities. Managing these conditions can reduce patients' morbidity and/or prevent further visual loss.

ii. The high prevalence of ocular abnormalities in stroke patients should stimulate a study on how much these abnormalities contribute to their disabilities. This information will indicate if stroke management regimens should include visual function enhancement.

\section{Acknowledgments}

None.

\section{Conflicts of interest}

Authors declare no conflicts of interest.

\section{References}

1. Thomas T, Stephen B, Mathers C. The global burden of cerebrovascular disease. 2006;1-2.

2. Easton JD, Saver JL, Albers GW, et al. Definition and Evaluation of Transient Ischemic Attack. Stroke. 2009;40(6):2276-2293.

3. Wahab K. The burden of stroke in Nigeria. Int J Stroke. 2008;3(4):290 292.

4. Fisher M, Norrving B. International Agenda for Stroke. American Heart Association's W.H.O. 2011.

5. Thrift AG, Thayabaranathan T, Howard G, et al. Global stroke Statistics. Int J stroke. 2017;12():13-32. 
6. Adeloye D. An Estimate of the Incidence and Prevalence of Stroke in Africa. PLoS One. 2014;9():100724.

7. American Stroke Association, A. heart A. Together to end Stroke. 2015.

8. Harrison T. In Harrison's principles of Internal Medicine. Longo D. et al, editors, 3810 The McGraw-Hill Companies; 2012.

9. Ogun SA, Ojini FI, Ogungbo B, et al. Stroke in South West Nigeria Stroke. 2005;36(6):1120-1122.

10. Danesi M, Okubadejo N, Ojini F. Prevalence of stroke in an urban mixed-income community in Lagos, Nigeria. Neuroepidemiology. 2007;28(4):16-223

11. Sanya EO, Desalu OO1, Adepoju F, et al. Prevalence of stroke in three semi-urban communities in middle-belt region of Nigeria: a door to door survey. Pan Afr Med J. 2015;20:4594.

12. Philip-Ephraim EE, Eyong KI, Chinenye S, et al. The Burden of Inpatient Neurological Disease in a Tropical African Hospital. Can J Neurol Sci. 2013;40(4):576-579.

13. Philip-Ephraim E, Eyong KI, Oparah SK, et al. Profile of Neurologica Emergencies At The Accidemt \& Emergency Department of A Tertiary Hospital in South Nigeria. J Neurol Sci Turk. 2013;30(1):72-80.

14. Duane T. Duane's Ophthalmology on CD ROM. In: Abegg M, Jason J, editors. Lippincott, Williams \& Wilkins. 2011.

15. National Geographic Channel. Facts About Perception. 2011.

16. AAO. In Basic and Clinical Science Cours. In: Cantor L, Rapuano C Cioffi G, editors. American Academy of Ophthalmologists. 111-126.

17. Duane T. In Duane's Ophthalmology on CD ROM. IN: Joel S, Romano J, editors. Wolters Kluwer Health/Lippincott, Williams \& Wilkins, 2014

18. American Academy. In Basic and Clinical Science Course. In: Cantor L Rapuano C, editors. AAO, 2014;155-159.

19. Feldman B, Kim J, Koushik T, et al. Hyperyensive Retinopathy. Eye Wiki. 2016.
20. Sight savers, I., NPPB, ICEH \& CBM. The Nigeria national blindness and visual impairment survey. (2007).

21. Olubor OJ, Uhumwangho OM, Omoti AE. Ocular disorders in stroke patients in a tertiary hospital in Nigeria. Niger J Clin Pr. 2016;19(1):397400.

22. Rowe FJ, Wright D2, Brand D, et al. Profile of Gaze Dysfunction following Cerebrovascular Accident. ISRN Ophthalmol. 2013;10:8-16.

23. Chang YS, Choi JE1, Kim SW, et al. Prevalence and associated factors of facial palsy and lifestyle characteristics: data from the Korean National Health and Nutrition Examination Survey 2010-2012. BMJ Open. 2016;6(11):12628.

24. Averbuch-Heller L, Leigh R., Mermelstein V, et al. Ptosis in patients with hemispheric strokes. Neurology. 2002;58(4):620-624.

25. Besharati MR, Rastegar A, Shoja MR, et al. Prevalence of retinopathy in hypertensive patients. Saudi Med J. 2006;27(11):1725-1728.

26. Abah E, Obiako O, Mahmoud-Ajeigbe A. et al. Routine retinal examination in patients with acute stroke in Ahmadu Bello University Teaching Hospital, Zaria, Nigeria. Int J Nutr Pharmacol Neurol Dis. 2012;2(3)229-232.

27. Rowe FJ, Wright D, Brand D, et al. A Prospective Profile of Visual Field Loss following Stroke: Prevalence, Type, Rehabilitation, and Outcome. Biomed Res Int. 2013;719096.

28. De Silva DA, Liew G, Wong MC, et al. Retinal Vascular Caliber and Extracranial Carotid Disease in Patients With Acute Ischemic Stroke The Multi-Centre Retinal Stroke (MCRS) Study. Stroke. 2009;40(12):36953699 .

29. Kawasaki R1, Wang JJ, Aung T, et al. Prevalence of Age-Related Macular Degeneration in a Malay Population. The Singapore Malay Eye Study. Ophthalmology. 2008;115(10):1735-1741.

30. Nwosu S. Age-related macular degeneration in Onitsha, Nigeria. Niger J Clin Pr. 2011;14(3):327-331. 